\title{
1 A novel transposable element based authentication protocol for Drosophila cell lines
}

3 Daniel Mariyappa ${ }^{1, *}$, Douglas B. Rusch ${ }^{2,}$, Shunhua Han ${ }^{3}$, Arthur Luhur ${ }^{1}$, Danielle Overton ${ }^{1,4}$,

4 David F. B. Miller ${ }^{2}$, Casey M. Bergman ${ }^{3,5}$, Andrew C. Zelhof ${ }^{1 \dagger}$

${ }^{1}$ Drosophila Genomics Resource Center, Biology Department, Indiana University, Bloomington, IN

${ }^{2}$ Center for Genetics and Bioinformatics, Biology Department, Indiana University, Bloomington, IN

$8{ }^{3}$ Department of Genetics and Institute of Bioinformatics, University of Georgia, Athens, GA

$9 \quad{ }^{4}$ Current address: Biology Department, Indiana University Purdue University Indianapolis, Indianapolis, IN

$10{ }^{5}$ Department of Genetics, University of Georgia, Athens, GA

$11 *$ Equal contribution

$12{ }^{\dagger}$ Corresponding author

\section{Abstract}

15 Drosophila cell lines are used by researchers to investigate various cell biological phenomena. It

16 is crucial to exercise good cell culture practice. Poor handling can lead to both inter- and

17 intraspecies cross-contamination. Prolonged culturing can lead to introduction of large- and

18 small-scale genomic changes. These factors, therefore, make it imperative that methods to

19 authenticate Drosophila cell lines are developed to ensure reproducibility. Mammalian cell line

20 authentication is reliant on short tandem repeat (STR) profiling, however the relatively low STR

21 mutation rate in D. melanogaster at the individual level is likely to preclude the value of this

22 technique. In contrast, transposable elements (TE) are highly polymorphic among individual flies

23 and abundant in Drosophila cell lines. Therefore, we investigated the utility of TE insertions as

24 markers to discriminate Drosophila cell lines derived from the same or different donor

25 genotypes, divergent sub-lines of the same cell line, and from other insect cell lines. We

26 developed a PCR-based next-generation sequencing protocol to cluster cell lines based on the

27 genome-wide distribution of a limited number of diagnostic TE families. We determined the

28 distribution of five TE families in S2R+, S2-DRSC, S2-DGRC, Kc167, ML-DmBG3-c2, mbn2,

29 CME W1 Cl.8+, and OSS Drosophila cell lines. Two independent downstream analyses of the

30 NGS data yielded similar clustering of these cell lines. Double-blind testing of the protocol

31 reliably identified various Drosophila cell lines. In addition, our data indicate minimal changes

32 with respect to the genome-wide distribution of these five TE families when cells are passaged

33 for at least 50 times. The protocol developed can accurately identify and distinguish the 
numerous Drosophila cell lines available to the research community, thereby aiding reproducible Drosophila cell culture research.

\section{Introduction}

As of 2018, the estimated of the number of publications using all cell culture studies is 2 million (BAIROCH 2018). However, problems with reproducibility and authenticity hamper their use (ALMEIDA et al. 2016). Poor culture practices in individual laboratories has led to many cases of inter- and intraspecies cross-contamination (CAPES-DAVIS et al. 2010). Additionally, prolonged passaging can lead to large- and small-scale genomic changes due to in vitro evolution that cause sub-lines of the same cell line to vary among laboratories (BEN-DAVID et al. 2018; LIU et al. 2019). For example, extensive passaging (>50 passages) of viral-transformed human lymphoblastoid cell lines is associated with increased genotypic instability $(\mathrm{OH}$ et al. 2013). Likewise, long term passaging of mammalian cell lines is known to lead to increased single nucleotide variations (PAVLOVA et al. 2015), reduced differentiation potential (YANG et al. 2018) and changes in the karyotype (WENGER et al. 2004). To overcome these inconsistencies in experiments across laboratories when using human cell lines, the American National

51 Standards Institute and the American Type Culture Collection (ANSI/ATCC ASN-002) have

52 provided a standard for vertebrate cell culture work. Moreover, the NIH offers guidelines for

53 authenticating key research resources that have been endorsed by several major journals 54 (ATCC 2011; NIH 2015).

Though most of above-mentioned problems and solutions relate to mammalian cell culture practice, a significant number of laboratories use Drosophila cells for basic research.

57 Drosophila cell lines are used by researchers to investigate a myriad of cellular processes 58 including receptor-ligand interactions (OZKAN et al. 2013), cellular signaling (ALBERT AND BOKEL 59 2017), circadian biology (ALBERT AND BOKEL 2017), metal homeostasis (MOHR et al. 2018), 60 cellular stress response (AGUILERA-GOMEZ et al. 2017), neurobiology (TSUYAMA et al. 2017), 61 innate immunity (NONAKA et al. 2017), and functional genomics (ALBERT AND BOKEL 2017), as 62 well as being used extensively for gene editing by CRISPR Cas9 technology (LUHUR et al. 63 2018). Furthermore, as part of the modENCODE project, the transcriptional and chromatin 64 profiles of a large panel of Drosophila cell lines were determined to facilitate studies on gene 65 function and expression (CHERBAS et al. 2011; KHARCHENKO et al. 2011). However, currently 66 there are no protocols available to authenticate Drosophila cell lines. In addition, the effects of 67 long-term passaging on Drosophila cell lines have not been formally investigated despite 
evidence for extensive changes from wild-type ploidy and copy number in many Drosophila cell lines (ZHANG et al. 2010; LEE et al. 2014), implying that insect cells can potentially exhibit genomic changes in culture like their mammalian counterparts.

Human cell line authentication guidelines recommend short tandem repeat (STR)

72 profiling as the method of choice for routine cell typing, although approaches using genomic

73 techniques yield more comprehensive information (ALMEIDA et al. 2016). The use of STR

74 profiling as the preferred method to authenticate human cell lines is based on high STR allelic

75 diversity among the donors for different cell lines, relatively low cost, stability of using STR

76 markers, and the historical availability of methods to assay STR variants during the

77 development of human cell line authentication protocols. There are a number of limitations with

78 the STR approach. The ANSI/ATCC ASN-002 standard for typing human cell lines with STRs is over 100 pages long and requires careful implementation for proper interpretation. Moreover, STR-based methods for human cell line authentication are primarily designed to discriminate cell lines derived from different donors, but are less powerful for discriminating cell lines or sublines from the same donor genotype.

Development of cell line authentication protocols requires understanding the genome biology of a species, the specific characteristics of the most widely used cell lines in that research community, and how these features can be used to leverage cost-effective modern genomic technologies. In Drosophila, the majority of widely-used cell lines have been derived from a limited number of donor genotypes. Coupled with the low STR mutation rate in Drosophila relative to humans (SCHUG et al. 1997), the use of STR profiling for discriminating different Drosophila cell lines is likely to be limited. In contrast, it is well-established that transposable elements (TE) are highly polymorphic among individual flies (CHARLESWORTH AND LANGLEY 1989) and that Drosophila cell lines have an increased TE abundance relative to whole flies (POTTER et al. 1979). These properties, together with the large number of potential insertion sites across the genome and stability of TE insertions at individual loci, suggest that TE insertions should theoretically be useful markers to simultaneously discriminate Drosophila cell lines made from different donor genotypes as well as from the same donor genotype, including divergent sub-lines of the same cell line. HAN et. al (2021) recently tested this prediction and

97 demonstrated that genome-wide TE insertion profiles can reliably cluster different Drosophila

98 cell lines from the same donor genotypes and discriminate cell lines from different donor 99 genotypes, while also preserving information about the laboratory of origin. A minimal subset of 100 six active TE families (297, copia, $m d g 3$, $m d g 1$, roo and 1731) was also determined to have 101 essentially the same discriminative power as the genome-wide dataset (HAN et al. 2021). 
Based upon these findings, we investigated if the genome-wide distribution of these six TE families could form the basis for a reliable protocol to authenticate Drosophila cell lines. As noted earlier, several of the modENCODE cell lines are extensively used to study genomic and cell biological processes (CHERBAS et al. 2011; KHARCHENKO et al. 2011). These cell lines are also amongst the most widely-ordered cell lines from Drosophila Genomics Resource Center (DGRC). Therefore, we used six modENCODE lines derived from various D. melanogaster developmental stages: S2R+, S2-DRSC, Kc167 (embryonic origin); ML-DmBG3-c2 (L3 larval CNS origin); mbn2 (larval circulatory system origin); and CME W1 Cl.8+ (wing disc origin) in our analysis. Two other non-modENCODE cell lines - S2-DGRC and OSS (ovarian somatic sheath) - that are ordered frequently from the DGRC were also included.

Here we present data supporting the utility of a genomic TE distribution (gTED) protocol

113 to authenticate $D$. melanogaster cell lines. The developed gTED protocol was able to generate

114 distinct TE genomic distribution signatures for all the cell lines tested. Moreover, using the gTED

115 protocol we were able to authenticate blinded samples from the Drosophila research

116 community, thus validating the protocol. Moreover, the gTED signatures of up to 50 passages of

117 S2R + cells do not cluster in a passage-dependent manner, indicating that this protocol could be 118 used to authenticate cell lines with up to 50 passages. Moving forward, we aim to expand the 119 repertoire of cell lines assessed for their TE genomic distribution. We now have a protocol that can be adopted by the Drosophila research community to authenticate their cell lines and provide the necessary standards as per NIH guidelines.

\section{Materials and Methods}

Drosophila cell lines and genomic DNA extraction

126 Our protocol development included six modENCODE lines derived from various Drosophila 127 developmental stages: embryonic - S2R+ (DGRC \#150, CVCL_Z831), S2-DRSC (DGRC \#181, 128 CVCL_Z992), Kc167 (DGRC \#1, CVCL_Z834); L3 larval CNS origin - ML-DmBG3-c2 (DGRC 129 \#68, CVCL_Z728); larval circulatory system origin - mbn2 (DGRC \#147, CVCL_Z706); and wing 130 disc origin - CME W1 Cl.8+ (DGRC \#151, CVCL_Z790) (Table 1). Two other non-modENCODE 131 cell lines - S2-DGRC (DGRC \#6, CVCL_TZ72) and OSS (ovarian somatic sheath, DGRC \#190, 132 CVCL_1B46), were also included in the protocol development phase. The S2R+, S2-DRSC, S2133 DGRC, mbn2 cells were cultured in the Shields and Sang M3 medium (Sigma, Cat\#: S8398) 134 supplemented with 10\% fetal bovine serum (FBS, Hyclone, GE Healthcare), bactopeptone 135 (Sigma) and yeast extract (Sigma) M3+BPYE+10\%FBS. ML-DmBG3-c2 cells were cultured in 
$136 \mathrm{M} 3+\mathrm{BPYE}+10 \%$ FBS with $10 \mu \mathrm{g} / \mathrm{ml}$ insulin (Sigma-Aldrich) while CME W1 Cl.8+ cells

137 required $\mathrm{M} 3+2 \% \mathrm{FBS}+5 \mu \mathrm{g} / \mathrm{ml}$ insulin $+2.5 \%$ fly extract containing medium. OSS cells were

138 cultured in M3 + 10\% FBS + 10\% fly extract with $60 \mathrm{mg}$ L-glutathione (Sigma-Aldrich, Cat\#:

$139 \mathrm{G} 6013$ ) and $10 \mu \mathrm{g} / \mathrm{ml}$ insulin (Sigma-Aldrich, Cat\#: 19278). Kc167 cells were cultured in CCM3

140 medium (Hyclone, Cat\#: SH30061.03). To extract total genomic DNA, cells were cultured to

141 confluency, harvested by pipetting, centrifuged and washed once with phosphate-buffered

142 saline (PBS). Genomic DNA (gDNA) was extracted from the PBS washed pellet using the Zymo

143 Quick-DNA ${ }^{\text {TM }}$ MiniprepPlusKit (Cat\#: D4068/4069), using 1 column for every 10 million cells.

144 Genomic DNA was generated for triplicate samples of all cell lines in order to investigate the

145 reproducibility of our protocol as well as to detect and mitigate potential mislabeling of individual

146 samples during the project.

\section{Blinded samples}

149 External blinded samples from eight cell lines were obtained as triplicates of frozen genomic 150 DNA samples extracted from insect cell lines from Dr. Sharon Gorski, British Columbia Cancer 151 Research Centre, Vancouver, Canada and the Drosophila RNAi Screening Center, Harvard

152 University (Table 2). The identities of the external samples sent to DGRC were blinded by the 153 sample donors. For internal blinded samples, genomic DNA was extracted from three cell lines 154 in triplicate (Table 2). The identities of the internal samples were blinded from the team 155 members involved in library preparation and downstream analyses. Genomic DNA for both the 156 external and internal blinded samples was extracted as per the protocol described above. The 157 team members involved in library preparation and downstream analyses were blind to the 158 identity and replicates of each sample.

\section{Passage experiment}

$161 \mathrm{~S} 2 \mathrm{R}+$ cells were plated at $1 \times 10^{6}$ cells per $\mathrm{ml}$ at every passage. A single passage experiment 162 was performed wherein cells were passaged every 2-3 days and replicates of the passages 163 were frozen at the $1^{\text {st }}, 10^{\text {th }}, 20^{\text {th }}, 30^{\text {th }}, 40^{\text {th }}$ and $50^{\text {th }}$ passages with the cell concentrations 164 between $2.5-8.6 \times 10^{6}$ cells per ml. Triplicate genomic DNA samples from each passage was 165 extracted as described above.

\section{Primer design}

168 Six TE families shown by HAN et. al (2021) to be sufficient to identify Drosophila cell lines based 169 on WGS data were used as initial candidates for primer design. These six TE families are all 
170 long terminal repeat (LTR) retrotransposons, which insert as full-length elements containing an 171 identical LTR that provides a reliably known junction for PCR at each terminus of the TE 172 (SMUKOWSKI HEIL et al. 2021). Primer design was based upon the protocol outlined in Figure 1, 173 involving a two-step PCR (Reaction A/B and Reaction A/B Nest PCR). Each step required one 174 primer to be within the TE at either end (one for Reaction $A$ at the 5 ' of the TE and one for 175 Reaction $B$ at the 3' of the TE). Additionally, primers for Reaction $A / B$ and Reaction $A / B$ Nest 176 PCR needed to have low similarity. Based on these requirements, the general workflow for 177 designing PCR primers for six diagnostic TE families for the eight focal cell lines was as follows:

1) Generate consensus sequences for LTRs of candidate TE families.

179 a. Whole genome sequencing (WGS) data from (ZHANG et al. 2010; LEE et al. 2014) and (HAN et al. 2021) for all focal cell lines were mapped against TE canonical sequences and merged into a single BAM file.

b. Variants were called on the merged BAM file and a VCF file was generated using bcftools call (v1.9).

c. Full length consensus sequences for all six TE families from VCF file was generated using bcftools consensus (v1.9) with variable sites encoded as ambiguities.

d. Both the 5' and 3' LTRs from the full-length TE consensus sequence for each family were extracted.

2) Detect the first round of primer candidates.

Primers for nested PCR were detected with primer3 (v2.5.0) (https://github.com/primer3org/primer3) using the following parameters:

PRIMER_LIBERAL_BASE $=1$; PRIMER_MAX_NS_ACCEPTED $=1$; PRIMER_NUM_RETURN=10; PRIMER_GC_CLAMP=1; PRIMER_DNA_CONC=25; PRIMER_SALT_MONOVALENT=50; PRIMER_MIN_TM=60; PRIMER_OPT_TM=62; PRIMER_MAX_TM=65; PRIMER_SALT_DIVALENT=2; PRIMER_DNTP_CONC=0; PRIMER_TM_FORMULA=1 PRIMER_OPT_SIZE=22; $\quad$ PRIMER_MIN_SIZE=18; PRIMER_MAX_SIZE=25; PRIMER_MIN_GC=40; PRIMER_MAX_GC=60; PRIMER_PRODUCT_SIZE_RANGE=75100 150-250 100-300 301-400 401-500 501-600 601-700 701-850 851-1000.

3) Detect the second round of non-overlapping primer candidates 
The same parameters as in the previous round of primer design were used, with the additional specification that the primers designed in the first round were added to a "mispriming library" to exclude these regions for primer prediction in the second round of primer candidates.

4) Finalize primers from both rounds of primer candidates The final primers for Reaction A/B PCR and Reaction A/B Nest PCR were selected from the

Final adjustments to the primer locations were made based on testing the respective primer pairs. The full list of primers used in the study are listed in Table S1.

213 Nextera libraries were constructed for all the genomic DNA samples by using Nextera DNA Flex 214 Library Prep Kit (Illumina, Cat\#: 20018705) (Figure 1A). Then, the Nextera libraries were diluted 215 into $1 \mathrm{nM}$, and $5 \mu \mathrm{l}$ of each was used as the template for the TE library construction. To amplify 216 the fragments with the TE-specific genomic context, two separate multiplex PCRs were 217 performed (Reactions $A$ and $B$, Figure 1B) using TE-specific primers for all six families 218 simultaneously in combination with the Illumina i5 primer. For Reactions $A$ and B, two sets of 219 primers (Forward and Reverse) were designed within the two LTRs of each of the TEs as 220 detailed above. Since the generation of the Nextera library is not direction specific, DNA 221 fragments can orient in either direction with respect to the i5 adaptor thus allowing for detection 222 at either ends of the TE by amplification with the Illumina i5 primer with a TE-specific primer. 223 Therefore, this PCR step amplified the DNA fragments containing the 5' (Reaction A, Reverse 224 primer) or 3' (Reaction B, Forward primer) flanking regions of the TEs. A second nested PCR was performed to enrich for the TE-genomic DNA junctions, utilizing nested primers from within 226 the Reactions A and B with the i5 adaptor (Figure 1C). Both Nest PCR primers contained a 227 specific overhang region (5' GTTCAGACGTGTGCTCTTCCGATCT 3') to facilitate addition of 228 the index in the next PCR step. The final step was the Index PCR, which was performed to add 229 the i7 adaptor and index by using the kit NEBNext® Multiplex Oligos for Illumina (cat: 6609S). 230 Briefly, equal volumes of the products of Reactions A and B Nest PCRs containing either the TE 2315 ' and 3' flanking regions were combined and used as the template. The Index PCR was 
232 performed by using the Illumina i5 primer and the NEBNext® Multiplex Oligos to add i7 adaptor

233 and index (Figure 1D). Finally, the TE libraries were constructed with both i5 adaptors (added by

234 Nextera library construction), i7 adaptors and indexes (added by the Index adding PCR).

\section{Protocol:}

236 Step 1:

237 - Nextera libraries are made by following standard protocol.

238 - Each library is diluted to $1 \mathrm{nM}$.

239

240 Step 2: Reaction A/B (Two sets of reactions)

242 Reaction A: Primers: i5 + TE Reaction A Rev (To amplify the 5' flanking region of TE gene)

243 Reaction B: Primers: i5 + TE Reaction B For (To amplify the 3' flanking region of TE gene)

244

245

2.1 PCR reagents:

5X Phusion buffer $\quad 10 \mu \mathrm{l}$

$100 \mathrm{mM}$ dNTP mix $\quad 0.5 \mu \mathrm{l}$

100 uM i5 Primer $\quad 0.5 \mu \mathrm{l}$

100 uM Reaction A/B (Rev/For) $0.5 \mu \mathrm{l}$

Phusion polymerase $\quad 0.5 \mu \mathrm{l}$

$1 \mathrm{nM}$ Library $\quad 5.0 \mu \mathrm{l}$

$\mathrm{dd}_{2} \mathrm{O} \quad 33 \mu \mathrm{l}$

Total $50 \mu \mathrm{l}$

2472.2 PCR settings:

$98^{\circ} \mathrm{C} \quad 30 \mathrm{sec}$

\begin{tabular}{lll}
\hline $98^{\circ} \mathrm{C}$ & $10 \mathrm{sec}$ & \\
$65^{\circ} \mathrm{C}$ & $30 \mathrm{sec}$ & $10 \mathrm{cycles}$ \\
$72^{\circ} \mathrm{C}$ & $60 \mathrm{sec}$ & \\
\hline $722^{\circ} \mathrm{C}$ & $5 \mathrm{~min}$
\end{tabular}

$72^{\circ} \mathrm{C} \quad 5 \mathrm{~min}$

$4^{\circ} \mathrm{C} \quad$ Hold 
2492.3 Cleaned with 0.9X AMPure XP beads, washed with $80 \%$ ethanol twice, and elute with $40 \mu \mathrm{l}$

250 Elution Buffer (EB).

251

252 Step 3: Nest PCR (Two sets of reactions)

253

254 Set 1: Primers: i5 + TE Reaction A Nest PCR Reverse (Template: Reaction A products)

255 Set 2: Primers: i5 + TE Reaction B Nest PCR Forward (Template: Reaction B products)

256

257 3.1 PCR reagents:

5X Phusion buffer $\quad 10 \mu \mathrm{l}$

$100 \mathrm{mM}$ dNTP mix $\quad 0.5 \mu \mathrm{l}$

100 uM i5 Primer $\quad 0.5 \mu \mathrm{l}$

100 uM NestPCR Reaction A/B (Rev/For) $0.5 \mu \mathrm{l}$

Phusion polymerase $\quad 0.5 \mu \mathrm{l}$

Reaction A/B products $\quad 38 \mu \mathrm{l}$

Total $50 \mu \mathrm{l}$

258

259 3.2 PCR setting:

260

$98^{\circ} \mathrm{C} \quad 30 \mathrm{sec}$

$98^{\circ} \mathrm{C} \quad 10 \mathrm{sec}$

$65^{\circ} \mathrm{C} \quad 30 \mathrm{sec} \quad 10$ cycles

$72^{\circ} \mathrm{C} \quad 60 \mathrm{sec}$

$72^{\circ} \mathrm{C} \quad 5 \mathrm{~min}$

$4^{\circ} \mathrm{C} \quad$ Hold

2623.3 Cleaned with 0.9X AMPure XP beads, wash with 80\% ethanol twice, and eluted with $19 \mu \mathrm{l}$

263 EB.

264

265 Step 4: Index adding PCR with NEBNext 6609 Primers

266

267

4.1 PCR reagents: 


\begin{tabular}{ll} 
5X Phusion buffer & $10 \mu \mathrm{l}$ \\
$100 \mathrm{mM}$ dNTP mix & $0.5 \mu \mathrm{l}$ \\
$100 \mathrm{uM}$ i5 Primer & $0.5 \mu \mathrm{l}$ \\
NEBNext 6609S Primer & $5 \mu \mathrm{l}$ \\
Phusion polymerase & $0.5 \mu \mathrm{l}$ \\
Nest PCR Reaction A + B products & $33.5 \mu \mathrm{l}$ \\
\hline Total & $50 \mu \mathrm{l}$
\end{tabular}

269

4.2 PCR settings:

271

\begin{tabular}{lll}
$98^{\circ} \mathrm{C}$ & $30 \mathrm{sec}$ & \\
\hline $98^{\circ} \mathrm{C}$ & $10 \mathrm{sec}$ & \\
$65^{\circ} \mathrm{C}$ & $30 \mathrm{sec}$ & 3 cycles \\
$72^{\circ} \mathrm{C}$ & $60 \mathrm{sec}$ & \\
\hline $72^{\circ} \mathrm{C}$ & $5 \mathrm{~min}$ &
\end{tabular}

$4^{\circ} \mathrm{C} \quad$ Hold

2734.3 Cleaned with 0.8X AMPure XP beads, washed with $80 \%$ ethanol twice, and eluted with $32 \mu \mathrm{l}$

274 of EB.

275

\section{Sequencing}

277 Paired end sequencing was performed on an Illumina NextSeq 500 with a 150-cycle midi-cycle 278 kits. The first read in a pair (Read 1, R1) corresponds to flanking genomic DNA; the second 279 read in a pair (Read 2, R2) corresponds to TE sequence. Raw sequencing data was submitted 280 to SRA (SRP323476).

Sample Processing and Transposable Element Identification

283 Reads were trimmed for adapters and low quality using Trimmomatic (v0.38; 284 ILLUMINACLIP:adapters.fa:3:20:6 LEADING:3 TRAILING:3 SLIDINGWINDOW:4:20 285 MINLEN:40). By design, R2 reads occur inside the TE and can be used to demultiplex individual 286 fragments by TE of origin from a multiplex PCR. To do this, R2 reads were aligned to a 287 database of the consensus sequences used for primer design of the relevant TEs using Bowtie2 288 (v2.3.5.1); the corresponding $\mathrm{R} 1$ reads from the same fragment were then demultiplexed into 
TE specific bins based on the best alignment of R2. R1 reads were then mapped with Bowtie2 (-local -k 2) to the complement and reverse-complement $D$. melanogaster genome (version 6.30) in which the TEs were N-masked (Figure 2; red plus green reads). Masking was performed by

292 searching consensus transposable elements sequences against the $D$. melanogaster genome

293 (version 6.30) using NCBI blastn (version 2.2.26) with the following parameters: -a 10 -e 1e-100

$294-F$ "m L" -U T -K 20000 -b 20000 -m 8. R1 reads that did not map with a uniquely best match to

295 the genome were subsequently excluded. Simultaneously, the R1 reads were mapped to the TE

296 consensus sequences. The initial goal was to identify any valid junction where we could

297 explicitly identify the transition from a unique genomic context into a TE, aka a TE junction

298 (Figure 2; green reads). For a R1 read to identify a junction, the local alignment to the genome

299 and the TE must be congruent such that the entire read was accounted for (+/- 2 bases). Valid

300 junctions were defined such that multiple independent reads with independent start sites in the

301 genome all identify the same breakpoint. To improve the sensitivity, all the data from all the

302 different samples was combined for junction identification. A valid junction had to have at least

30312 reads with 4 distinct start positions. Once the junctions were identified, 300 bp of genomic

304 sequence outside and juxtaposed to the TE junction were isolated, which would include either 5'

305 or 3' or both ends of the inserted TE (Figure 2).

\section{Clustering and Visualization}

308 Read datasets were analyzed in their entirety or by random sub-sampling using vsearch 309 (v2.14.2) (ROGNES et al. 2016) down to 10 million reads, in order to control for sequencing depth 310 and explore how many reads were necessary per cell line to produce reliable results. Read 311 counts from sub-sampled datasets mapped to dm6 in the $300 \mathrm{bp}$ intervals adjacent to TE 312 junctions defined above were used to generate a binary matrix indicating the presence/absence 313 of the TEs in any given sample. This binary matrix was constructed with custom code based on 314 the observation that there are either many reads or very few reads per sample for any given TE 315 insertion site. After normalizing the number of TE associated reads per sample, a z-score was 316 calculated for every TE across the samples. Positive z-scores were assigned as present and 317 negative z-scores as absent. Because z-score normalization uses the mean of a sample, if all or 318 most of the samples are positive, by definition, half of the samples would end up with a negative 319 z-score. To avoid this mis-identification of positive samples, we add a dummy zero value to the 320 set of samples for every real sample included before z-score calculation. This data was then 321 visualized in $\mathrm{R}$ using the gplots function heatmap.2. The identities of blinded samples were 
322 estimated based on the clustering of these samples within the dendrogram derived from known

323 samples.

325 Code

326 Code and notes on running the TE detection and clustering pipeline are available at: 327 https://github.com/mondegreen/DrosCellID.git.

\section{Results}

Drosophila cells have distinct TE signatures

332 Previous analysis of available whole genome sequencing (WGS) data revealed that genomic TE distribution can reliably cluster cell lines based on their genotype and laboratory of origin (HAN et al. 2021). Moreover, WGS analysis using a limited set of six TE families (297, copia, mdg3, $m d g 1$, roo and 1731) was sufficient to replicate the clustering observed when data from all TE families was used (HAN et al. 2021). Nevertheless, an alternative approach that selectively enriches the six TE families would be more efficient and cost-effective. Therefore, based on these analyses, here we set out to determine if targeted identification of the genomic distribution of a small number of diagnostic TE families could be used to 1) to build an authentication platform for Drosophila cell lines based on unique genomic TE distribution (gTED) signatures for each cell line, 2) test the validity of this protocol by assessing the identities of blinded samples, both internal and those provided by the Drosophila community and 3) assess if cell lines subjected to extensive passaging retain the unique cell-specific gTED signatures.

To achieve these goals, we developed a novel TE based NGS enrichment protocol described in the Materials and Methods (Figure 1). Briefly, this protocol uses a multiplexed nested PCR approach to selectively amplify the library elements containing the 5' and 3' ends of the target TE families (Reaction $A$ and $B$, Figure 1). The products from the final PCR amplification step were subjected to next generation sequencing (NGS) and downstream analyses to determine the type of TE and identify the unique genomic DNA flanking the TE sequence.

The NGS data obtained was first used to identify TE junctions using the bioinformatic

352 strategy outlined in Figure 2. Since the number of reads observed upon amplification with mdg3353 specific primers was very low, mdg3 was excluded from further analyses. Normalized counts of 354 reads mapping near TE junctions for the remaining five families were then used to hierarchically 355 cluster all the cell lines. Reads mapping close to the identified TE junctions, whether at 5' or 3' 
end or both, were included in further analyses (Figure 2). The resulting dendrogram showed that the triplicate samples from most cell lines clustering together (Figure 3). Upon processing the NGS data using an alternative approach (Supplementary File 1), a comparable clustering of all the samples was observed (Figure S1). In both approaches, one replicate each from S2 DGRC (S2-DGRC_2) and S2 DRSC (S2-DRSC_2) did not cluster with the other replicates from these cell lines (Figure 3, Figure S1). The similar clustering from both bioinformatic approaches suggests the non-conforming clustering of these two replicates is not an artifact of genomic or computational methods, and was most likely caused by reciprocal sample mislabeling during gDNA extraction. Regardless of the cause of these two discrepancies, the majority of samples (2/3) for both S2 DGRC and S2 DRSC are respectively consistent with one another, providing confidence in the identity of these cell line clusters.

Distinct gTED signatures, a composite of the five TE families assessed, were observed for every cell line investigated (Figure 3 and Figure S2). The tree visualization heatmap demonstrates that there are very few shared TE insertions between all cell lines (Figure 3). In general, the total number of TEs detected by this technique was higher in embryonic cell lines as opposed to cell lines derived from larval or adult tissues (Table 1, Figure S2). The total number of TEs mapped was similar for the replicates of each of the cell lines as seen in the UpSET plot (LEX et al. 2014) for these samples (Figure S2). For many of the cell lines, the majority of TE insertions detected were unique relative to those shared with other cell lines. For example, OSS replicates have 262 unique TEs that are not found in any other cell line investigated, with $\leq 9$ TEs in common with any other individual cell lines (Figure S2). The only lines that do not conform to having majority unique TE insertions are S2 DGRC and S2 DRSC as they share a considerable proportion of the TEs with S2R+ (Figure S2). Nevertheless, unique patterns of gTED were sufficient to distinguish between the various S2 sublines (Figures 3, S1 and S2). Two of the three larval tissue derived cell lines (ML-DmBG3-c2, mbn2 and CME W1 $\mathrm{Cl} .8+$ ) have fewer genomic TE insertions as compared to embryonic S2 and Kc167 lines. However, mbn2, a cell line reportedly derived from the larval circulatory system (GATEFF 1977; GATEFF et al. 1980) has a gTED signature very close to those of the S2 lines, which are all of hematopoietic origin (SCHNEIDER 1972). The unexpected similarity between S2 lines and mbn2 was also described recently by Han et al. (2021) based on WGS based TE distribution analysis.

386 These analyses demonstrated that the protocol developed to determine genomic distribution of 387 a set of five TE families in Drosophila cell lines can be utilized to create unique cell line-specific 388 signatures. 
TE signatures of Drosophila cell lines can be employed for authentication

To assess the value of the developed gTED pipeline and validate it, we next queried if the cell line-specific gTED signatures could be employed to determine the identities of blinded samples (Table 2). The blinded samples were either donations from the Drosophila community (external samples) or generated internally at DGRC. All blinded samples, as well as triplicates of an internal control for S2R+ (DGRC_Blinded_control_1-3), were processed as outlined in the Materials and Methods section.

Of the eight external cell lines processed from two different donating labs, six robust gTED signatures were obtained (Figure S3A). However, very few TE insertions detected in six samples, possibly from two cell lines (Figure S3A). gTED profiles for three samples (DRSC_Blinded_13-15) was very similar to the internal control from S2R+ processed in this run (DGRC_Blinded_control_1-3, Figure S3A). For fifteen of the eighteen samples with robust gTED profiles, clusters of triplicates were observed, indicating that each cluster possibly represents replicates samples of five cell lines (Figures 4 and S3A). One sample did not cluster distinctly with any of the other samples (SGLab_Blinded_4, Figures 4 and S3A), however this sample had a gTED profile that is visually most similar to samples SGLab_Blinded_5-6 (Figure S3A). The six samples that had very few TE insertions (triplicates for each labelled DRSC_Blinded_4-6 and DRSC_Blinded_7-9) each passed the genomic DNA and library preparation quality control steps, and the consistent lack of TE insertions among replicates suggested that this was a reproducible signal. Upon clustering the external blinded samples with the previously characterized set of TE signatures it was possible to predict the identities of these samples (Figure 4, Table

2) as DRSC_Blinded_1-3 and DRSC_Blinded_10-12 (Kc167), DRSC_Blinded_4-6 and DRSC_Blinded_7-9 (No identification), DRSC_Blinded_13-15 (S2R+), DRSC_Blinded_16-18 (S2), SGLab_Blinded_1-3 (mbn2) and SGLab_Blinded_5-6 (S2). Moreover, the clustering generated with gTED has the resolution to identify the various $\mathrm{S} 2$ sublines. For instance, it is evident that DRSC_Blinded_13-15 are closest to S2R+, DRSC_Blinded_16-18 to S2-DGRC, and SGLab_Blinded_5-6 to S2-DRSC (Figure 4). The

417 investigators who donated the external samples confirmed that the identities determined by the 418 gTED protocol was accurate for all the samples as predicted (Table 2). The two cell lines with 419 very few TE insertions for which a cell line identity prediction could not be generated were 420 mosquito cell lines (Figure 4, Table 2). These experiments demonstrated that the gTED protocol 421 could reliably identify blinded Drosophila samples submitted to DGRC by the community.

422 All three internal blinded cell lines had unique gTED signatures that clustered distinctly 423 relative to all previously-characterized gTED signatures (Figures 4 and S3B). Nevertheless, the 
424 triplicates from each of the internal blinded cell lines reliably clustered together (Figure 4). Upon

425 unblinding (Table 2), the internal blinded samples were found to be from three cell lines not

426 included in the initial development phase of the project: 1182-4H (DGRC_Blinded_A, 427 DGRC\#177, CVCL_Z708), Ras[V12];wts[RNAi] (DGRC_Blinded_B, DGRC\#189, CVCL_IY71) 428 and delta_I(3)mbt-OSC (DGRC_Blinded_C, DGRC\#289). Thus, processing blinded samples 429 through the gTED pipeline revealed that 1) reliable identification of samples with known gTED 430 signatures can be achieved, 2) the protocol is capable of distinguishing Drosophila versus non431 Drosophila cell lines and 3) D. melanogaster cell lines previously uncharacterized by the gTED 432 protocol can be identified as such, without providing a false identification.

TE signature of $S 2 R+$ is retained upon extensive passaging

435 Extensive passaging of cell lines can potentially alter cellular genomes (WENGER et al. 2004; $\mathrm{OH}$ 436 et al. 2013). Apart from gross genomic changes, extensive passaging introduced single 437 nucleotide polymorphisms in mammalian cell lines (PAVLOVA et al. 2015). To determine the 438 effect of extensive passaging on the gTED signatures generated in this study, we passaged 439 S2R + cell line 50 times and isolated genomic DNA in triplicate at every tenth passage for 440 processing (Fig. 5A). Upon generating a cluster using the gTED protocol, it is evident that the 441 triplicates from the passages cluster randomly and not according to passage numbers (Fig. 5B).

442 Moreover, all replicates from every passage tested form a distinct cluster (Fig. S4) indicating 443 that extensive passaging of $S 2 R+$ does not alter the $S 2 R+g T E D$ signature for up to 50 444 passages.

\section{Discussion}

448 The aim of this study was to develop and test a cell authentication protocol that could reliably 449 identify the most commonly used Drosophila cell lines to help researchers validate their 450 reagents as per the $\mathrm{NIH}$ mandate. Our novel protocol allowed us to define unique gTED 451 signatures that could identify each of the Drosophila cell lines that were tested here. In addition, 452 the resolution obtained from the gTED signatures allows for distinguishing between S2 sublines. 453 Data presented here demonstrate that the gTED signatures of the replicates of most cell lines 454 cluster together, outlining the reproducibility of the gTED protocol while also underscoring the 455 value of having replicate samples for reliable cell line identification. Crucially, accurate 456 identification of blinded samples donated by the research community validated the gTED 457 protocol in a real-world setting. 
To reliably identify a $D$. melanogaster cell line using the gTED protocol, an established gTED signature is a prerequisite. Towards this end, we have now established gTED signatures for the widely distributed lines, S2R+, S2 DGRC, S2 DRSC, Kc167 and ML-DmBG3-c2 lines (LUHUR et al. 2018). In addition, gTED signatures are also available for OSS, mbn2, CME W1

$462 \mathrm{Cl} .8+, 1182-4 \mathrm{H}$, Ras[V12];wts[RNAi] and delta I(3)mbt-OSC lines. Importantly, the lack of an 463 established gTED signature does not lead to misidentification, as was observed with the internal 464 blinded samples. In the event that a cell line without an established gTED signature needs to be 465 authenticated, a stock from the DGRC repository with the same identity will be assayed 466 concurrently to serve as a control. In due course, DGRC will also expand the gTED protocol to 467 include as many cell lines from our repository as possible. These efforts will ensure the creation of a comprehensive database of gTED signatures for Drosophila cell lines.

Mosquito cell lines included as blinded samples helped clarify that the gTED protocol can discriminate non-Drosophila cell lines. In Ae. aegypti and An. gambiae, $10 \%$ and $6 \%$ of the total genome, respectively, is comprised of LTR retrotransposons (NENE et al. 2007; MELO AND WALLAU 2020). Presence of active LTR transposons, specifically Ty1/copia has also been described in Aag2 (Ae. aegypti) cells (MARINGER et al. 2017). Since we confirmed that the DNA and library preparation for these samples were comparable, it is most likely therefore that the TE-specific primers used in this study cannot amplify mosquito TE families. Our results demonstrate that in pure samples mosquito cells can be distinguished from $D$. melanogaster cell lines using the gTED protocol. However, detecting low levels of inter- or intra-species contamination might a more challenging pursuit. A D. melanogaster cell line contaminated with low levels of a mosquito cell line is unlikely to be detected with gTED, necessitating using other methods for such specific instances. A future avenue is to explore the sensitivity of the gTED protocol to intra- or inter-species contamination. In addition, it will be imperative to determine if we can determine low levels of contamination of Drosophila cell lines containing unique gTED signatures.

Our analysis also demonstrated that the genomic distribution of TEs is largely unchanged over 50 passages in S2R+cells. The narrow window into the passaging-associated 486 genomic structure provided by the gTED protocol is most likely not representative of more 487 complex genomic and/or transcriptomic changes that the extensively passaged cells might have 488 undergone. Nevertheless, S2R+ cells passaged continuously for up to 50 times can still be 489 identified with the gTED protocol. Among the $\mathrm{S} 2$ lines assessed in this study, it has been 490 proposed that the S2R+ line is possibly the closest to the original Schneider line (SCHNEIDER 491 1972; YANAGAWA et al. 1998). The other two S2 sublines, S2-DGRC and S2-DRSC, are isolates 
with less clear history from the original Schneider isolates before being added to the DGRC repository (AYER AND BENYAJATI 1992; CHERRY et al. 2005). All three of the S2 sublines assessed have unique gTED signatures that discriminate them and can be used to identify

495 blinded cell lines precisely to the S2 subline. In general, S2 sublines have a more complex TE-

496 landscape, higher aneuploidy and copy number variation than other $D$. melanogaster cell lines

497 (HAN et al. 2021). The possibility that the gTED signature can be used as a proxy for broader 498 genomic changes remains to be investigated.

In summary, utilizing the genomic distribution of five TE families we have developed the gTED pipeline to facilitate the authentication of Drosophila cell lines. We demonstrate that the developed gTED protocol can assign distinct signatures to the various Drosophila cell lines tested. Blinded and extensively passaged samples can now be authenticated employing the gTED protocol. Researchers working with Drosophila cell lines can independently authenticate cell lines being used in their laboratories using the protocol and code described in this study. Alternatively, DGRC will implement a cost-based service for the research community to access and authenticate their cell lines for both publications and research funding. Ultimately, our goal is to include more cell lines from the DGRC repository into the gTED pipeline and generate gTED signatures for all cell lines deposited with the DGRC.

Data availability

511 All data necessary for confirming the conclusions in this paper are included in this article and in 512 supplemental figures and tables. All the NGS data has been deposited at Sequence Read 513 Archive available with the accession number: SRP323476

\section{Acknowledgments}

516 We thank Dr. Kris Klueg for critical input in shaping the manuscript. We thank Grace Kim 517 (DRSC), Dr. Stephanie Mohr (DRSC), Nancy Erro Go (British Columbia Cancer Research 518 Centre) and Dr. Sharon Gorski (British Columbia Cancer Research Centre) for providing the 519 blinded genomic DNA samples. We thank Chunlin Yang, Jie Huang, and Sumitha Nallu at the 520 Center for Genomics and Bioinformatics (Indiana University, Bloomington) for their help with 521 NGS sample processing. This work was supported by NIH grants (5P40OD010949 and 522 3P40OD010949-16S2) to the Drosophila Genomics Resource Center, National Science 523 Foundation under Grant No. CNS-0521433 to Center for Genomics and Bioinformatics (Indiana 524 University, Bloomington) and the University of Georgia Research Foundation to CMB. 
Figure 1: Protocol used for generating libraries to establish genomic transposable element distribution signatures. A) Fragmented genomic DNA (gDNA; light brown lines) from the Nextera libraries containing TEs (green bar) and flanking gDNA were amplified with the randomly oriented i5 (blue arrow) and i7 (black arrow) primers. B) Reactions A and B involved

532 amplification with the i5 primer oriented in either direction with respect to the TE, in combination 533 either with TE-specific Reverse (dark brown arrow) and Forward (dark grey arrow) primers, 534 respectively. C) The Nest PCR reactions amplified from within the products of the respective 535 Reactions A and B using the i5 primer and either the TE-specific Nest Reverse (light brown 536 arrow) or TE-specific Nest Forward (light grey arrow) primers. Read 2 anchors were added onto 537 both the Nest PCR primers. D) The final amplification step was performed with the i5 primer and 538 the Read 2 anchor with the i7 index primer (black box). The reads from the genome sequences flanking the TE are designated as Read 1; the reads internal to the TE are designated Read 2.

Figure 2: Read mapping strategy used to generate genomic transposable element distribution signatures. Read 1 (R1) reads from demultiplexed fragments were used to identify the transposon junctions (green) from the set of all $\mathrm{R} 1$ reads. The schematic represents $\mathrm{R} 1$ reads at junctions on either end (5' or 3') of a TE. The number of reads that specifically identify a junction is relatively small compared to the total number of reads near the junction. Variation in sequencing depth and subtle differences in the insert sizes produced by the Nextera library could cause junctions to be missed if only explicit junction calls are used. To avoid these issues, after the junctions have been identified, a $300 \mathrm{bp}$ region of genomic sequence flanking the transposon is used to quantify the number of R1 reads (red) associated with that junction.

Figure 3: Clustering of cell lines based on genomic transposable element distribution.

552 The cell line clustering was derived upon processing NGS data as described in the Materials 553 and Methods. The triplicates for each cell lines are indicated with 1-3 following the cell line 554 name.

Figure 4: Cell line authentication of double-blind samples using genomic transposable

557 element distribution signatures. Triplicate samples of external blinded cell lines from the lab 558 of Dr. S. Gorski (shaded yellow) and Drosophila RNAi Screening Center (shaded green) along 559 with internal blinded samples (shaded brown) and internal control samples (shaded red) were 
processed with the gTED protocol (Figure 2B) and clustered as described in the Materials and Methods along with the previously processed known samples. The cell lines that the blinded samples cluster with are indicated with the black lines. Internal blinded samples cluster as a separate group. Samples DRSC_Blinded_4-9 with very few or no TEs detected were from mosquito cell lines (Table 2).

Figure 5: Genomic transposable element distribution signatures for S2R+ cells do not cluster by passage number. A) Schematic outlining the protocol to acquire samples between 1-50 S2R+ passages for assessment by the gTED protocol. B) Clustering of all the passage samples generated based on TE predictions. The triplicates samples of every passage are shaded in one color each.

Supplementary Figure 1: Clustering of cell lines based on genomic transposable element distribution using an alternative bioinformatics pipeline. The cell line clustering is derived from processing NGS data as described in Supplementary File 1. The triplicates for each cell lines are indicated with 1-3 following the cell line name.

Supplementary Figure 2: Unique TEs distinguish cell lines assessed by gTED. The UpSET plot. Filled in dots indicate the samples that share the particular set of TEs. The absolute number of TEs for each of the samples is plotted as Set Size. internal (B) blinded samples assessed using the gTED protocol have unique gTED signatures that cluster replicates by cell identity.

Supplementary Figure 4: S2R+ cells retain unique gTED signature despite extensive passaging. All samples from this study assessed using the gTED protocol indicate that all the $\mathrm{S} 2 \mathrm{R}+$ passages cluster together, still retaining a unique cell-line specific gTED signature. The $\mathrm{S} 2 \mathrm{R}+$ passages are shaded in green. 
593 this alternative approach for cell lines used in the development phase of the project is shown in

594 Supplementary Figure 1.

595

596 Supplementary File 2: Table of samples ID listed in SRA accession used for gTED

597 analysis. The 75 samples used for the analysis in the manuscript are listed in the table. The

598 other 39 samples listed in SRP323476 were used for testing and development.

599

600 Supplementary File 3: Presence absence matrix for cell line clustering. The final data

601 matrix used for cell line clustering is available at:

602 https://github.com/mondegreen/DrosCellID/blob/main/combined.presence-absence.example.tsv.

603 
NIH Rigor and Reproducibility: Principles and Guidelines for Reporting Preclinical Research and Endorsement by major journals., pp.

Aguilera-Gomez, A., M. Zacharogianni, M. M. van Oorschot, H. Genau, R. Grond et al., 2017 Phospho-Rasputin Stabilization by Sec16 Is Required for Stress Granule Formation upon Amino Acid Starvation. Cell Rep 20: 2277.

Albert, E. A., and C. Bokel, 2017 A cell based, high throughput assay for quantitative analysis of Hedgehog pathway activation using a Smoothened activation sensor. Sci Rep 7: 14341.

Almeida, J. L., K. D. Cole and A. L. Plant, 2016 Standards for Cell Line Authentication and Beyond. PLoS Biol 14: e1002476.

ATCC, 2011 Authentication of Human Cell Lines: Standardization of STR Profiling., pp. in ANSI/ATCC ASN-0002-2011. ANSI.

Ayer, S., and C. Benyajati, 1992 The binding site of a steroid hormone receptor-like protein within the Drosophila Adh adult enhancer is required for high levels of tissue-specific alcohol dehydrogenase expression. Molecular and Cellular Biology 12: 661-673.

Bairoch, A., 2018 The Cellosaurus, a Cell-Line Knowledge Resource. J Biomol Tech 29: 25-38.

Ben-David, U., B. Siranosian, G. Ha, H. Tang, Y. Oren et al., 2018 Genetic and transcriptional evolution alters cancer cell line drug response. Nature 560: 325-330.

Capes-Davis, A., G. Theodosopoulos, I. Atkin, H. G. Drexler, A. Kohara et al., 2010 Check your cultures! A list of cross-contaminated or misidentified cell lines. Int J Cancer 127: 1-8.

Charlesworth, B., and C. H. Langley, 1989 The population genetics of Drosophila transposable elements. Annu Rev Genet 23: 251-287.

Cherbas, L., A. Willingham, D. Zhang, L. Yang, Y. Zou et al., 2011 The transcriptional diversity of 25 Drosophila cell lines. Genome Res 21: 301-314.

Cherry, S., T. Doukas, S. Armknecht, S. Whelan, H. Wang et al., 2005 Genome-wide RNAi screen reveals a specific sensitivity of IRES-containing RNA viruses to host translation inhibition. Genes \& Development 19: 445-452.

Gateff, E., 1977 Malignant neoplasms of the hematopoietic system in three mutants of Drosophila melanogaster. Ann Parasitol Hum Comp 52: 81-83.

Gateff, E., L. Gissmann, R. Shrestha, N. Plus, H. Pfister et al., 1980 Characterization of two tumorous blood cell lines of Drosophila melanogaster and the viruses they contain. Invertebrate Systems in vitro: 517-533.

Han, S., P. J. Basting, G. Dias, A. Luhur, A. C. Zelhof et al., 2021 Transposable element profiles reveal cell line identity and loss of heterozygosity in Drosophila cell culture. Genetics: (In press).

Kharchenko, P. V., A. A. Alekseyenko, Y. B. Schwartz, A. Minoda, N. C. Riddle et al., 2011 Comprehensive analysis of the chromatin landscape in Drosophila melanogaster. Nature 471: 480-485.

Lee, H., C. J. McManus, D. Y. Cho, M. Eaton, F. Renda et al., 2014 DNA copy number evolution in Drosophila cell lines. Genome Biol 15: R70.

Lex, A., N. Gehlenborg, H. Strobelt, R. Vuillemot and H. Pfister, 2014 UpSet: Visualization of Intersecting Sets. IEEE Trans Vis Comput Graph 20: 1983-1992.

Liu, Y., Y. Mi, T. Mueller, S. Kreibich, E. G. Williams et al., 2019 Multi-omic measurements of heterogeneity in HeLa cells across laboratories. Nat Biotechnol 37: 314-322.

Luhur, A., K. M. Klueg and A. C. Zelhof, 2018 Generating and working with Drosophila cell cultures: Current challenges and opportunities. Wiley Interdiscip Rev Dev Biol: e339.

Maringer, K., A. Yousuf, K. J. Heesom, J. Fan, D. Lee et al., 2017 Proteomics informed by transcriptomics for characterising active transposable elements and genome annotation in Aedes aegypti. BMC Genomics 18: 101. 
654

655

656

657

658

659

660

661

662

663

664

665

666

667

668

669

670

671

672

673

674

675

676

677

678

679

680

681

682

683

684

685

686

687

688

689

690

691

692

693

694

695

696

697

698

Melo, E. S. d., and G. L. Wallau, 2020 Mosquito genomes are frequently invaded by transposable elements through horizontal transfer. PLOS Genetics 16: e1008946.

Mohr, S. E., K. Rudd, Y. Hu, W. R. Song, Q. Gilly et al., 2018 Zinc Detoxification: A Functional Genomics and Transcriptomics Analysis in Drosophila melanogaster Cultured Cells. G3 (Bethesda) 8: 631-641.

Nene, V., J. R. Wortman, D. Lawson, B. Haas, C. Kodira et al., 2007 Genome Sequence of Aedes aegypti, a Major Arbovirus Vector. Science 316: 1718-1723.

$\mathrm{NIH}, 2015$ Enhanced Reproducibility through Rigor and Transparency, pp. NIH.

Nonaka, S., Y. Ando, T. Kanetani, C. Hoshi, Y. Nakai et al., 2017 Signaling pathway for phagocyte priming upon encounter with apoptotic cells. J Biol Chem 292: 8059-8072.

Oh, J. H., Y. J. Kim, S. Moon, H. Y. Nam, J. P. Jeon et al., 2013 Genotype instability during long-term subculture of lymphoblastoid cell lines. J Hum Genet 58: 16-20.

Ozkan, E., R. A. Carrillo, C. L. Eastman, R. Weiszmann, D. Waghray et al., 2013 An extracellular interactome of immunoglobulin and LRR proteins reveals receptor-ligand networks. Cell 154: 228-239.

Pavlova, G. V., A. A. Vergun, E. Y. Rybalkina, P. R. Butovskaya and A. P. Ryskov, 2015 Identification of structural DNA variations in human cell cultures after long-term passage. Cell Cycle 14: 200-205.

Potter, S. S., W. J. Brorein, Jr., P. Dunsmuir and G. M. Rubin, 1979 Transposition of elements of the 412, copia and 297 dispersed repeated gene families in Drosophila. Cell 17: 415427.

Rognes, T., T. Flouri, B. Nichols, C. Quince and F. Mahe, 2016 VSEARCH: a versatile open source tool for metagenomics. PeerJ 4: e2584.

Schneider, I., 1972 Cell lines derived from late embryonic stages of Drosophila melanogaster. J Embryol Exp Morphol 27: 353-365.

Schug, M. D., T. F. Mackay and C. F. Aquadro, 1997 Low mutation rates of microsatellite loci in Drosophila melanogaster. Nat Genet 15: 99-102.

Smukowski Heil, C., K. Patterson, A. S.-M. Hickey, E. Alcantara and M. J. Dunham, 2021 Transposable Element Mobilization in Interspecific Yeast Hybrids. Genome Biology and Evolution 13.

Tsuyama, T., A. Tsubouchi, T. Usui, H. Imamura and T. Uemura, 2017 Mitochondrial dysfunction induces dendritic loss via elF2alpha phosphorylation. J Cell Biol 216: 815834.

Wenger, S. L., J. R. Senft, L. M. Sargent, R. Bamezai, N. Bairwa et al., 2004 Comparison of established cell lines at different passages by karyotype and comparative genomic hybridization. Biosci Rep 24: 631-639.

Yanagawa, S., J. S. Lee and A. Ishimoto, 1998 Identification and characterization of a novel line of Drosophila Schneider S2 cells that respond to wingless signaling. J Biol Chem 273: 32353-32359.

Yang, D., N. Li and G. Zhang, 2018 Spontaneous adipogenic differentiation potential of adiposederived stem cells decreased with increasing cell passages. Mol Med Rep 17: 6109-6115.

Zhang, Y., J. H. Malone, S. K. Powell, V. Periwal, E. Spana et al., 2010 Expression in aneuploid Drosophila S2 cells. PLoS Biol 8: e1000320. 


\begin{tabular}{|c|c|c|c|c|}
\hline Cell line & Tissue source & $\begin{array}{c}\text { DGRC Stock } \\
\text { Number }\end{array}$ & Cellosaurus ID & $\begin{array}{c}\text { Number of TE insertions } \\
\text { Mean (+/- SD) }\end{array}$ \\
\hline S2R+ & Embryo & 150 & CVCL_Z831 & $1009( \pm 30.4)$ \\
\hline S2 DGRC & Embryo & 6 & CVCL_TZ72 & $704( \pm 3.2)$ \\
\hline mbn2 & $\begin{array}{c}\text { Larval circulatory } \\
\text { system }\end{array}$ & 147 & CVCL_Z706 & $633( \pm 6.4)$ \\
\hline S2 DRSC & Embryo & 181 & CVCL_Z992 & $530( \pm 14.8)$ \\
\hline OSc167 & Embryo & 1 & CVCL_Z834 & $516( \pm 18.3)$ \\
\hline AME-W1-Cl.8+ & Larval wing disc & 151 & CVCL_Z790 & $404( \pm 8.5)$ \\
\hline ML-DmBG3-c2 & Larval CNS & 68 & CVCL_Z728 & $227( \pm 4.7)$ \\
\hline
\end{tabular}

Table 1: Summary of transposable element (TE) insertions detected by gTED. The total number TE insertions that were detected in each of the listed cell lines is presented as a mean $(n=3)$ of the samples analyzed. SD=standard deviation, CNS: Central Nervous System 


\begin{tabular}{|c|c|c|c|}
\hline Sample label & Source & $\begin{array}{l}\text { Identification with } \\
\text { gTED pipeline }\end{array}$ & $\underset{n}{\text { Confirmatio }}$ \\
\hline DRSC_Blinded_1-3 & DRSC & Kc167 & Kc167 \\
\hline DRSC_Blinded_4-6 & DRSC & No ID & A. $g$ \\
\hline DRSC_Blinded_7-9 & DRSC & No ID & A. $a$ \\
\hline DRSC_Blinded_10-12 & DRSC & Kc167 & Kc167 \\
\hline DRSC_Blinded_13-15 & DRSC & $\mathrm{S} 2 \mathrm{R}+$ & $\mathrm{S} 2 \mathrm{R}+$ \\
\hline DRSC_Blinded_16-18 & DRSC & S2 & S2 \\
\hline SGLab_Blinded_1-3 & $\begin{array}{l}\text { Gorski } \\
\text { Lab }\end{array}$ & mbn2 & $\mathrm{mbn} 2$ \\
\hline SGLab_Blinded_4-6 & $\begin{array}{c}\text { Gorski } \\
\text { Lab }\end{array}$ & S2 & $\mathrm{S} 2$ \\
\hline DGRC_Blinded_A & Internal & No ID & $1182-4 \mathrm{H}$ \\
\hline DGRC_Blinded_B & Internal & No ID & $\begin{array}{c}\text { Ras[V12];wts } \\
\text { [RNAi] }\end{array}$ \\
\hline DGRC_Blinded_C & Internal & No ID & $\begin{array}{c}\text { delta I(3)mbt- } \\
\text { OSC }\end{array}$ \\
\hline
\end{tabular}

Table 2: List of blinded samples processed. Blinded samples were donated by external (Drosophila RNAi Screening Center and Dr. S. Gorski) or generated internally. The identifications were made upon processing the sample through the genomic TE distribution pipeline followed by computational analysis. No ID: The genomic TE signatures of the cell lines did not match with any of the lines analyzed to provide a positive identification. $A$. a: cell line derived from Aedes aegypti; A. g: cell line derived from Anopheles gambiae. 
bioRxiv preprint doi: https://doi org/10.1101/2021.08 16.456580; this version posted August 17, 2021. The copyright holder for this preprint (which was not certified by peer review) is the author/funder, who has granted bioRxiv a license to display the preprint in perpetuity. It is made available under aCC-BY-NC-ND 4.0 International license.

A

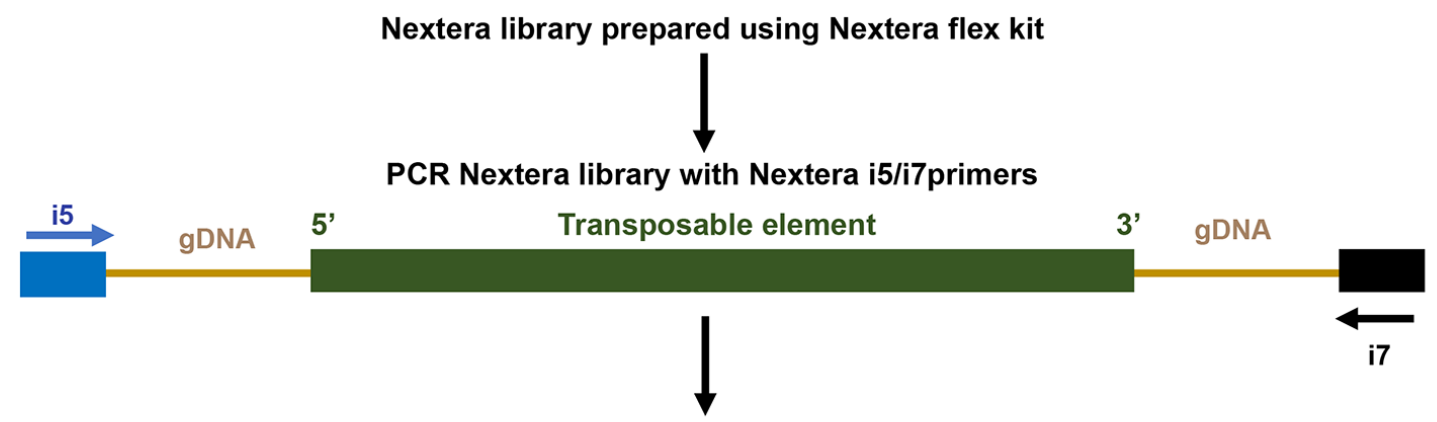

B

C

D

Random Nextera Library generation

Reaction A

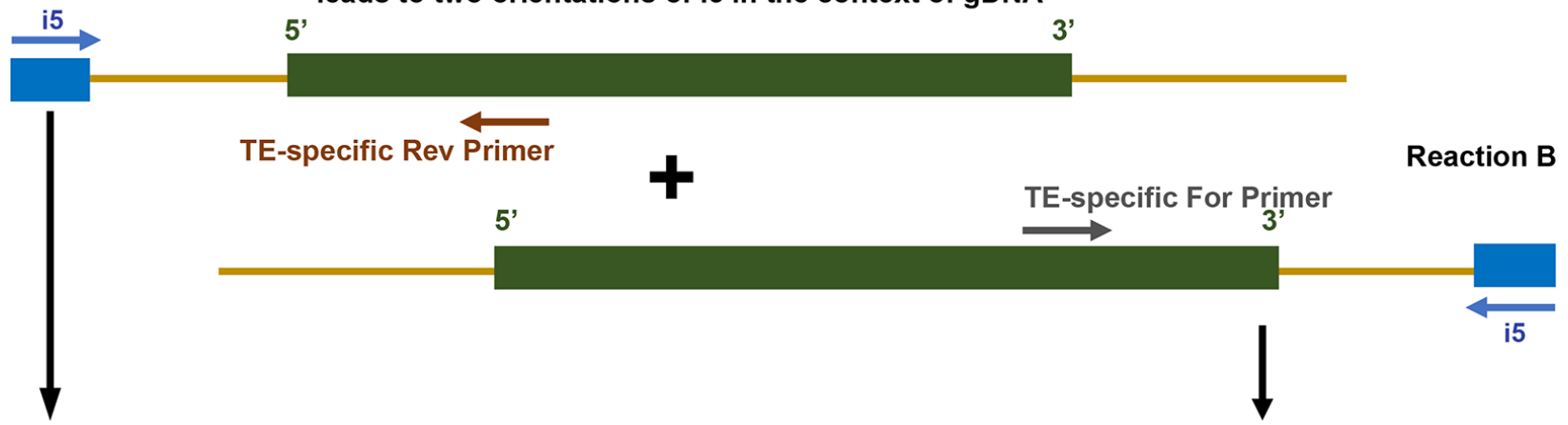
leads to two orientations of i5 in the context of gDNA

Reaction A Nest PCR

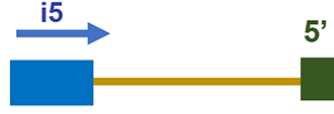

5
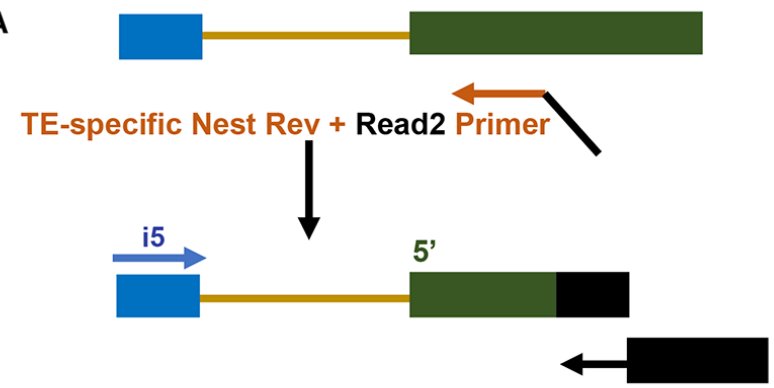

Read2 anchor + NEBNext 6609 i7 index primer
Figure 1

Reaction B Nest PCR

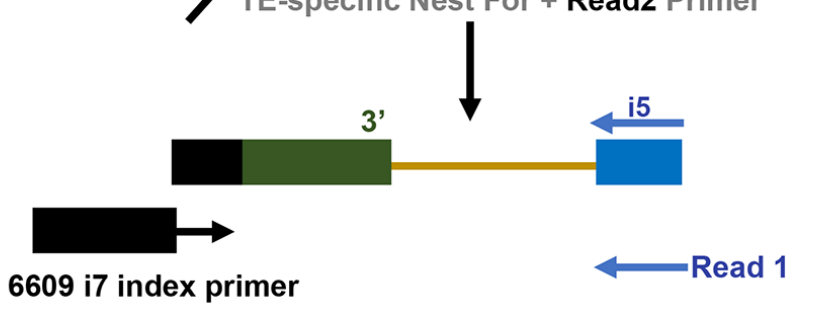


Figure 2

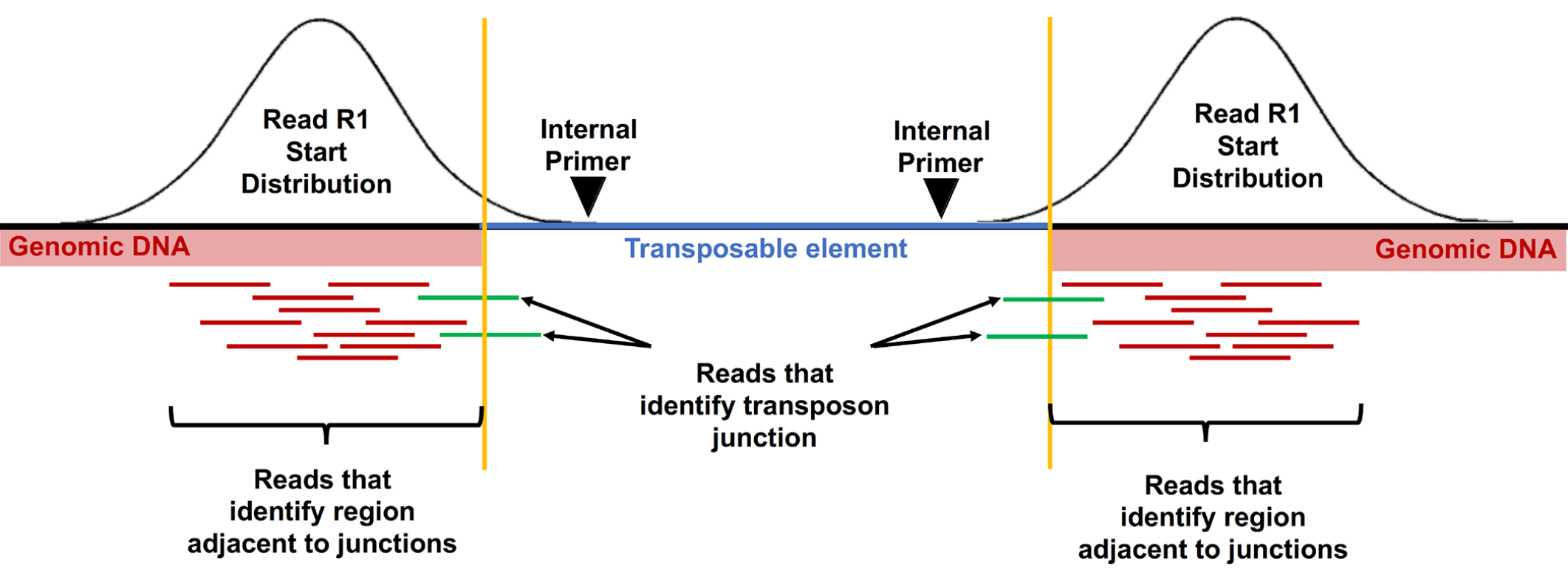


Figure 3

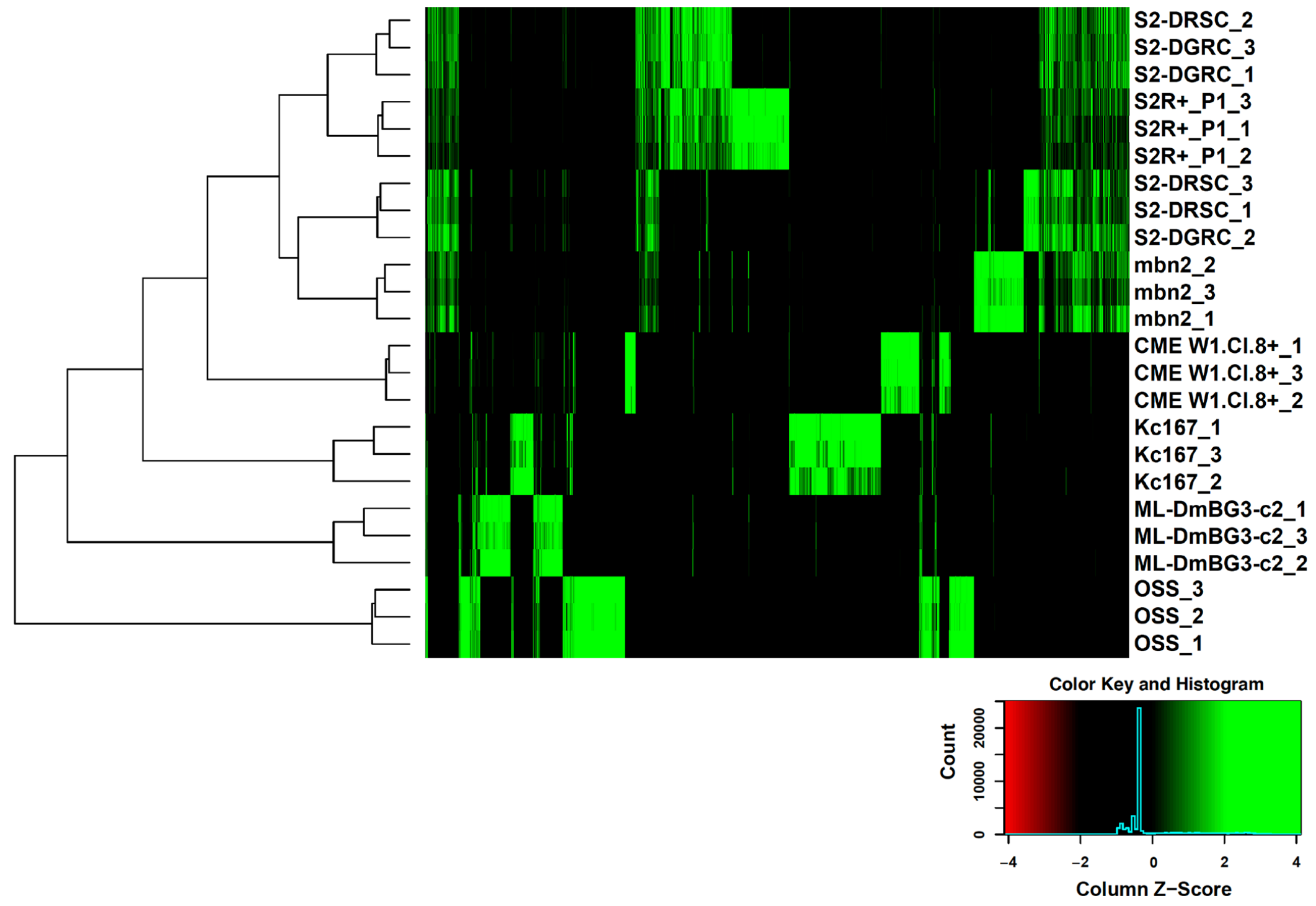


bioRxiv preprint doi: https://doi.org/10.1101/2021.08.16.456580; this version posted August 17, 2021. The copyright holder for this preprint (which was not certified by peer review) is the author/funder, who has granted bioRxiv a license to display the preprint in perpetuity. It is made available under aCC-BY-NC-ND 4.0 International license.

Figure 4

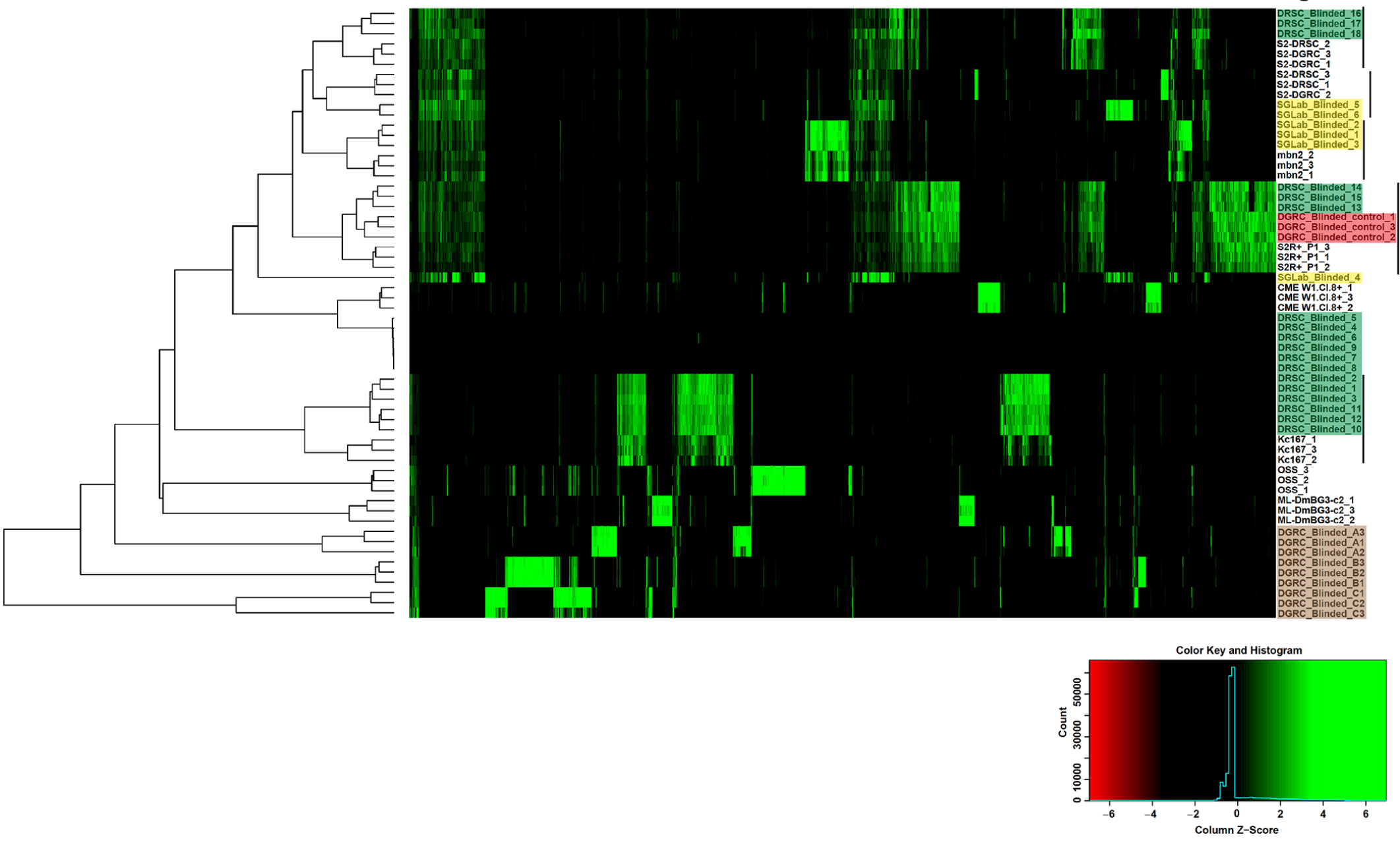




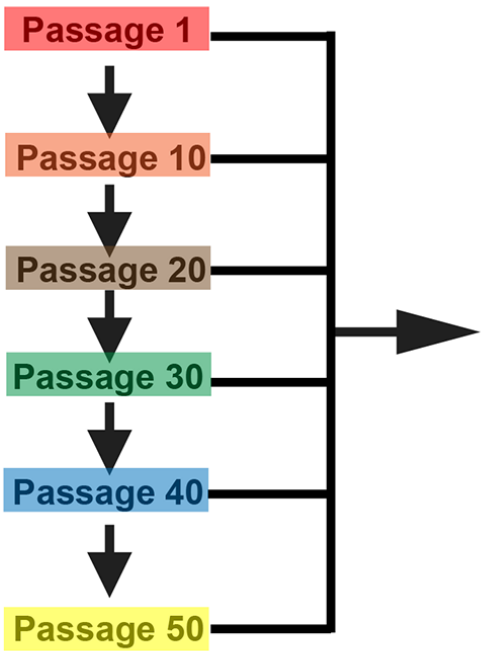

\section{Genomic DNA isolation from triplicates at every 10th passage}

Figure 5

\section{Run samples through NGS pipeline and downstream analysis to determine genome-wide TE distribution}

B

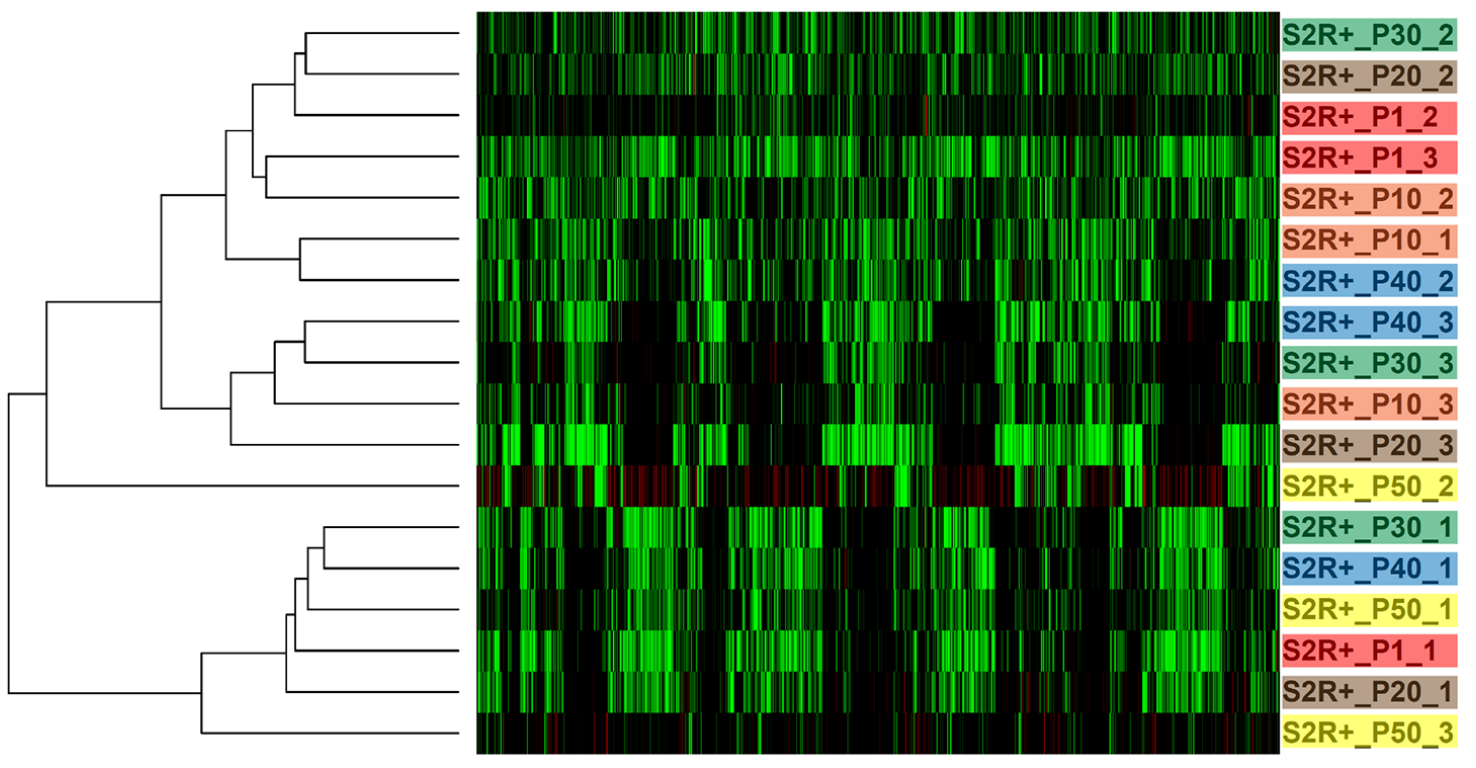

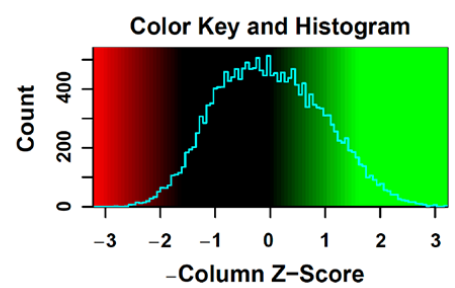

